\title{
Preparation of experimental measurements using a firearm
}

\author{
Michal Gracla ${ }^{1 *}$, Lukáš Pavlík² \\ ${ }^{1}$ Tomas Bata University in Zlin,, Faculty of Applied Informatics, Namesti T.G.Masaryka 5555, 76000 Zlin, Czech Republic \\ ${ }^{2}$ Tomas Bata University in Zlin,, Faculty of Applied Informatics, Namesti T.G.Masaryka 5555, 76000 Zlin, Czech Republic
}

\begin{abstract}
In this paper, we focus on one specific firearm, which is air gun, and further on preparation of experimental measurements. In the introduction, it is discussed what other science teams investigate and why. Moreover, it is mentioned a ballistics protection of an individual and, consequently, the justification of why other science teams rather use other firearms than on which we focus. Then, we describe used aids for given experimental measurements. Finally, descriptions and results from our experimental measurements with above mentioned aids are discussed.
\end{abstract}

\section{Introduction}

Firearms and ballistic protection of an individual are linked closely. The only protection against firearms is a ballistic protection of an individual eventually solid obstacles e.g. reinforced concrete or brick walls. Similar as any ballistic protection, while using bigger caliber than on which it is made, it is possible to shoot through. This can be seen on ballistic protection tests. Each class of ballistic protection has its own caliber against which is tested. In the Czech Republic, it is standard C CN 39 5360 - Resistance tests of protective means - Technical requirements and testing. However, the American standard 0101.04 is more widespread in the world. In the Czech Republic, this American standard is more widespread and when choosing ballistic protection, the designation is rather in accordance with this American standard instead of the Czech one. Ballistic protection and its testing have been studied in [1-5].

Ballistic protection of an individual and firearms are investigated by many research and professional teams. These teams also deal with the different directions and its components. This is illustrated by the following examples $[6,7]$. However, each team deals only with firearms or cool weapons and protection against them. Just few teams deal with other weapons such as gas weapons or even mechanical weapons. In most cases, these firearms are included in so-called free-to-sales stores from the age of 18 . Their muzzle velocity is not great enough to kill in most cases. Even so, they are firearms, which can cause very serious fire injuries or even death in the wrong hands.

In this work, we do not deal with these firearms and resistances, but rather we look for ways to compare them with other firearms classified in the Czech Republic as weapons of category D. Although, weapons of category D are freely available in the Czech Republic since 18, they are still firearms, so they have to be treated as such. In order to deal with these firearms, there is no need for a certified shooting range, but only a safe place, so as not to damage third parties or injure the uninvolved people. This is the main reason why we chose these firearms for testing. In future publications, our intention is to find out if there is any coefficient that can predict the effect of our "safe" firearms in comparison with "dangerous" firearms. First, however, the starting conditions must be the same for both experimental tests. In this paper, we deal with this problem.

\section{Methods and measurements}

The necessary equipment was prepared for the experiments. In particular, a firearm classified into weapons of category D according to Czech Act No. 119/2002 Coll., on firearms and ammunition, was included in this equipment. Specifically, it was an air gun TEX 086. Additionally, enough ammunition had to be earmarked for this firearm, chronograph to measure velocity of bullets and, last but not least, capture materials (modelling mass and absorbent cotton wool). Supporting equipment was the digital scale (PLT 20003DM from KERN) for determining the masses of diabolo pellets and steel balls of various sizes.

Before performing any experiment with firearms, not only weapons of category $\mathrm{D}$, the used equipment and other equipment needed for experiments should be tested first. As a weapon of category D, the air gun (using compressed gas or air for shooting a projectile) was selected. The function of an air gun in general is derived from immediate release of compressed air or other gas (usually $\mathrm{CO}_{2}$ ) which has low temperature. For air guns, the projectiles are released with maximum kinetic energy of $16 \mathrm{~J}$. This is the main difference between air gun and firearms. In this particular case, it was an airgun on compressed gas (air gun) tagged TEX 086 (Figure 1). [8]

* Corresponding author: gracla@utb.cz

(C) The Authors, published by EDP Sciences. This is an open access article distributed under the terms of the Creative Commons Attribution License 4.0 (http://creativecommons.org/licenses/by/4.0/). 


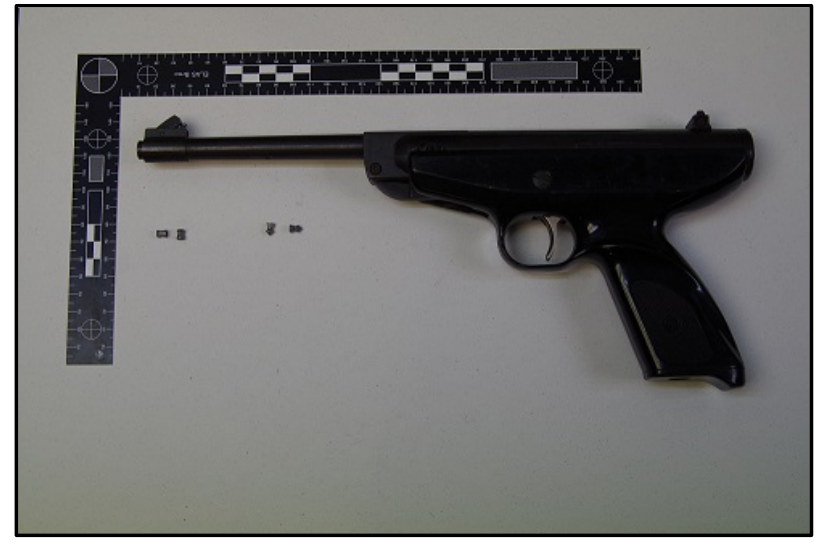

Fig. 1. Airgun model TEX 086.

This type of gun is widely used even by kids at camps or amusement parks. This pistol is a spring-piston air gun made in Czechoslovakia. Other parameters can be found in Table 1. The production was discontinued in the $80 \mathrm{~s}$.

Table 1. Basic parameters of TEX 086.

\begin{tabular}{|l|l|}
\hline Parameters & Value \\
\hline Type & pistol \\
\hline Length of piston & $346 \mathrm{~mm}$ \\
\hline Length of barrel & $185 \mathrm{~mm}$ \\
\hline Caliber & $4.5 \mathrm{~mm}(.177)$ \\
\hline Groove number & 12 \\
\hline Groove step & $450 \mathrm{~mm}$ \\
\hline Aiming line & $292 \mathrm{~mm}$ \\
\hline Mass of piston & $1.22 \mathrm{~kg}$ \\
\hline Speed of projectile & $90 \mathrm{~m} . \mathrm{s}^{-1}$ (Diabolo pellets) \\
\hline Trigger pull adjustable & max. 3.72 N \\
\hline Cocking strength & max. $11.77 \mathrm{~N}$ \\
\hline
\end{tabular}

The model uses cocking (Figure 2) to pump the piston, which compresses the spring. After pulling the trigger, the piston is released which pressure the air behind the pellet until it is propelled forward.

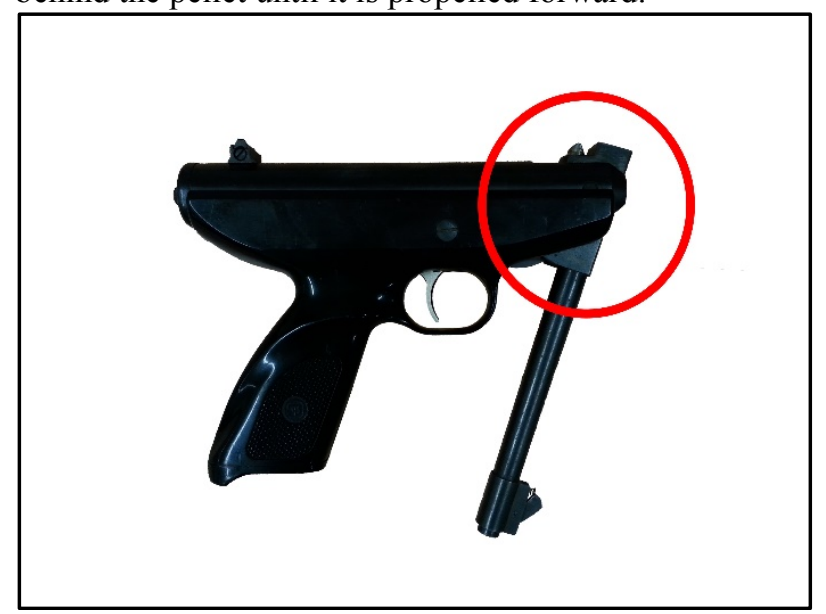

Fig. 2. Principle of cocking weapon.

During experimental testing, one type of ammunition was used. This ammunition was a diabolo pellets called HUNTER IMPACT (Figure 3).

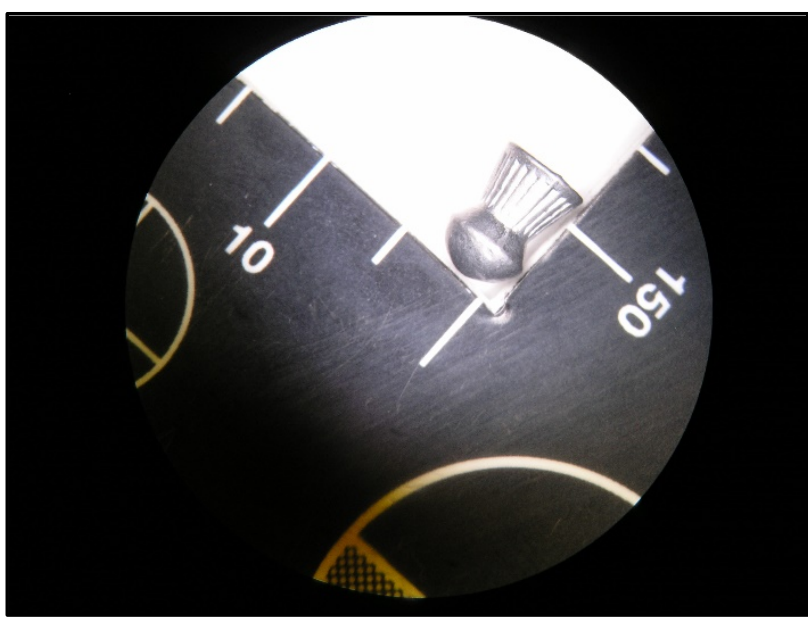

Fig. 3. Microscopic photo of used diabolo pellet, model HUNTER IMPACT.

Characteristic parameter of diabolo pellet can be seen in Table 2. The size was measured with digital calliper (accuracy $0.01 \mathrm{~mm}$ ) and the weight was measured with digital scale PLT 2000-3DM from KERN on 30 randomly picked diabolo pellets to ensure compliance.

Table 2. Parametrs of used diabolo pellet model HUNTER IMPACT.

\begin{tabular}{|l|l|}
\hline Parameters & Value \\
\hline Producer & GAMO \\
\hline Caliber & $4.5 \mathrm{~mm}(.177)$ \\
\hline Weight & $0.49 \mathrm{~g}(7.56 \mathrm{gr})$ \\
\hline Measured length & $6.41 \pm 0.05 \mathrm{~mm}$ \\
\hline Measured width & $4.65 \pm 0.08 \mathrm{~mm}$ \\
\hline Measured weight & $0.49 \pm 0.01 \mathrm{~g}$ \\
\hline
\end{tabular}

The las device for experimental testing was the ballistic chronograph. This device is an electronic gate (Figure 4), from company Caldwell Chronograph, named Premium Kit with IR light. This chronograph was used for the measurements of diabolo pellet velocity. The electronic gate measures velocity in $\mathrm{m}_{\mathrm{s}} \mathrm{s}^{-1}$ in the range from 1.5 to $3,000 \mathrm{~m} \cdot \mathrm{s}^{-1}$ and also in $\mathrm{ft} . \mathrm{s}^{-1}$. It is most frequently used device to measure the velocity of bullets from firearms as well as arrows.

The basic experiments included checking the accuracy of chronograph. Further, it was about determination of loss of ammunition (diabolo pellet) after the barrel of air gun passed through. The following experiment served to determine the accuracy of the chronograph velocities and the second unverified chronograph. Finally, we verified the number of measurements required to avoid disturbing the efficiency and distorting the results of the experimental measurement. 


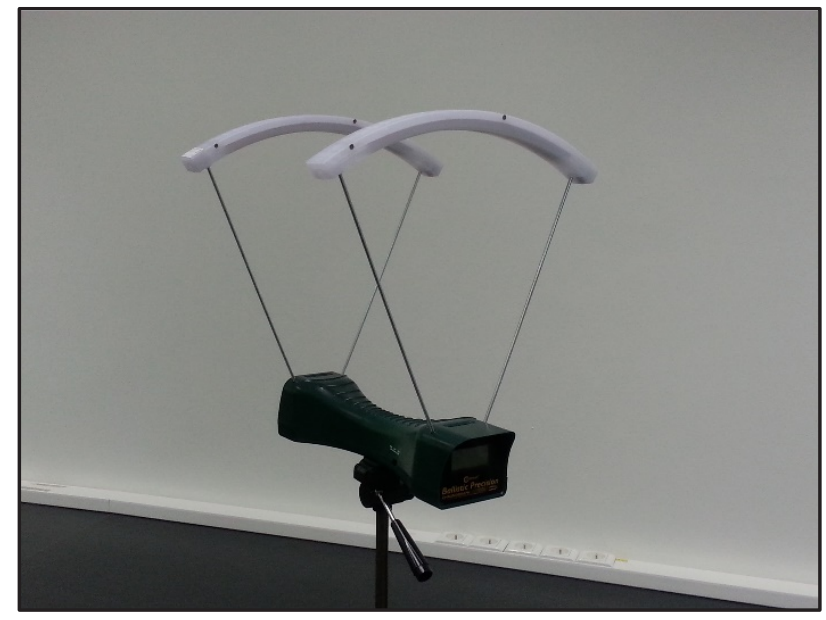

Fig. 4. Ballistic chronograph used to measure velocity of diabolo pellets.

\section{Results and discussion}

\subsection{Calibration of the chronograph}

In the first experiment, it was necessary to determine the accuracy of the chronograph Caldwell Premium Kit (Figure 4). The experiment was performed by throwing steel balls of three different sizes from two defined height levels through the above-mentioned electronic gates. The height was measured in advance and no initial velocity was previously given to the steel balls. Three steel balls were thrown ten times over the electronic gates. Previously, the theoretical velocity (using a mathematical relationship for free fall seen in equation 1) was calculated and the actual velocity was experimentally verified. The drawback of the entire experimental measurement was that the electronic gates can display the velocity only in whole numbers (min. Units $\mathrm{m} / \mathrm{s}$ ). The results of the mathematical calculation and can be seen in equation 2 and 3 .

The height was defined as $3.1 \mathrm{~m}$. From this height, the value of $0.47 \mathrm{~m}$ (the height of the last gate of electronic chronograph above the ground) was subtracted. From the mathematical relationship for free fall, the theoretical velocity of the handball steel balls was determined. The second height was $5.6 \mathrm{~m}$ with the same difference from the ground $0.47 \mathrm{~m}$. According to the same mathematical relationship, the second theoretical velocity was calculated.

$$
\begin{gathered}
v=\sqrt{2 g h} \\
v=\sqrt{2 * 9.81 *(3.1-0.47)} \\
=7.18 \mathrm{~m} / \mathrm{s} \\
v=\sqrt{2 * 9.81 *(5.6-0.47)} \\
=10.03 \mathrm{~m} / \mathrm{s}
\end{gathered}
$$

Where:

$\mathrm{v}-$ Velocity $[\mathrm{m} / \mathrm{s}]$,

$\mathrm{g}$ - Gravitational acceleration on Earth $\left[\mathrm{m} / \mathrm{s}^{2}\right]$,

$\mathrm{h}-$ Height $[\mathrm{m}]$.

At the first experiment, the theoretical speed was 0.2 $\mathrm{m} / \mathrm{s}$ lower than the measured one. The second experiment revealed the same measurement error, as can be seen from the graph (Figure 5)

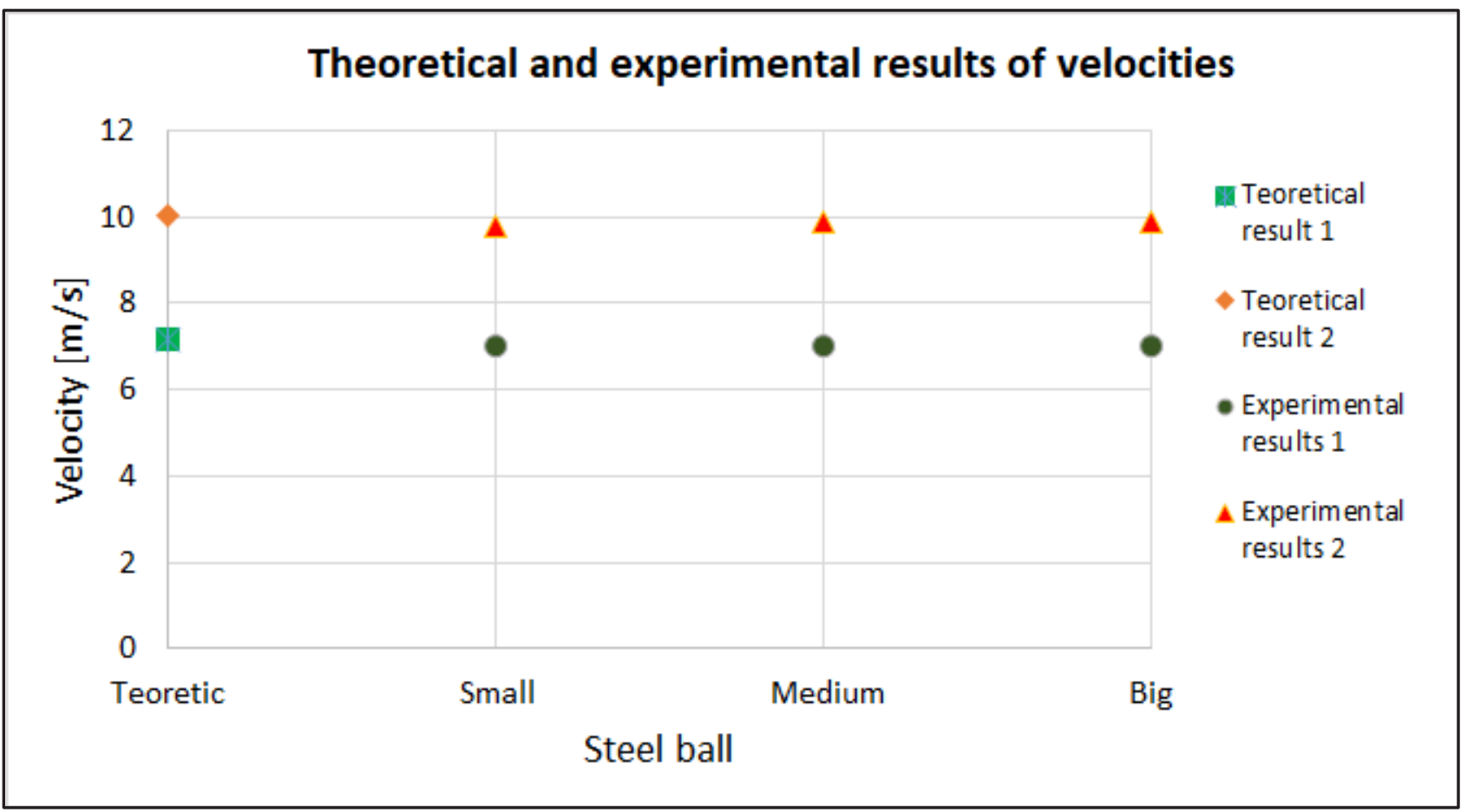

Fig. 5. Theoretical and experimental results of velocities. 
It means that the error in this measurement is systematic, due to the inability to measure the velocity more accurately. For firearms, however, this error is in tenth $\mathrm{m} / \mathrm{s}$ negligible, because it is not necessary to measure the velocity so accurately. It suffices to measure the speeds per unit $\mathrm{m} / \mathrm{s}$ for firearms. Therefore, these electronic gates are sufficient, even if they are unable to measure higher resolution of velocities than $\mathrm{m} / \mathrm{s}$ units.

\subsection{Loss of weight in the barrel}

The second experiment is important because of subsequent measurements (for example, to determine the velocities and momentum of bullets after penetration of the defined materials), and to answer the important question of whether the bullet mass is lost after the shoot in the barrel of firearm. The experiment was conducted on the air gun TEX 086 with diabolo pellet Hunter Impact. Various experiments were performed to eliminate the error.

The first part of this experiment was focused on determining the weight of each diabolic separately. The weight was determined before the shot, and then after pulling the diabolo pellet from the capture material. Two capture materials were used, modeling mass and absorbent cotton wool. In this experiment, the possibility of losing the weight of diabolo pellet when stopping in the catch material was not solved. This loss of weight was (if any) only solved when the diabolo pellet went through the barrel.

The second part of this experiment was similar. The difference was that diabolo pellets were not weighed separately, but they were weighed together. In all four experiments, 10 diabolo pellets were used. The results can be seen in Table 3 and Figure 6. For the measurements when the weight of the diabolo pellets was determined separately, the values were multiplied by the total number and already given for all 10 diabolo pellets.

Table 3. Ammunition weight.

\begin{tabular}{|l|c|c|}
\hline \multicolumn{1}{|c|}{ Capture material } & $\begin{array}{c}\text { Before } \\
\text { shooting }\end{array}$ & $\begin{array}{c}\text { After } \\
\text { shooting }\end{array}$ \\
\hline $\begin{array}{l}\text { Absorbent cotton wool - together (1 in } \\
\text { the Figure 6) }\end{array}$ & $4.885 \mathrm{~g}$ & $4.882 \mathrm{~g}$ \\
\hline $\begin{array}{l}\text { Absorbent cotton wool - on one's own } \\
(2 \text { in the Figure 6) }\end{array}$ & $4.869 \mathrm{~g}$ & $4.868 \mathrm{~g}$ \\
\hline $\begin{array}{l}\text { Modeling mass - together (3 in the } \\
\text { Figure 6) }\end{array}$ & $4.877 \mathrm{~g}$ & $4.875 \mathrm{~g}$ \\
\hline $\begin{array}{l}\text { Modeling mass - on one's own (4 in } \\
\text { the Figure 6) }\end{array}$ & $4.868 \mathrm{~g}$ & $4.863 \mathrm{~g}$ \\
\hline
\end{tabular}

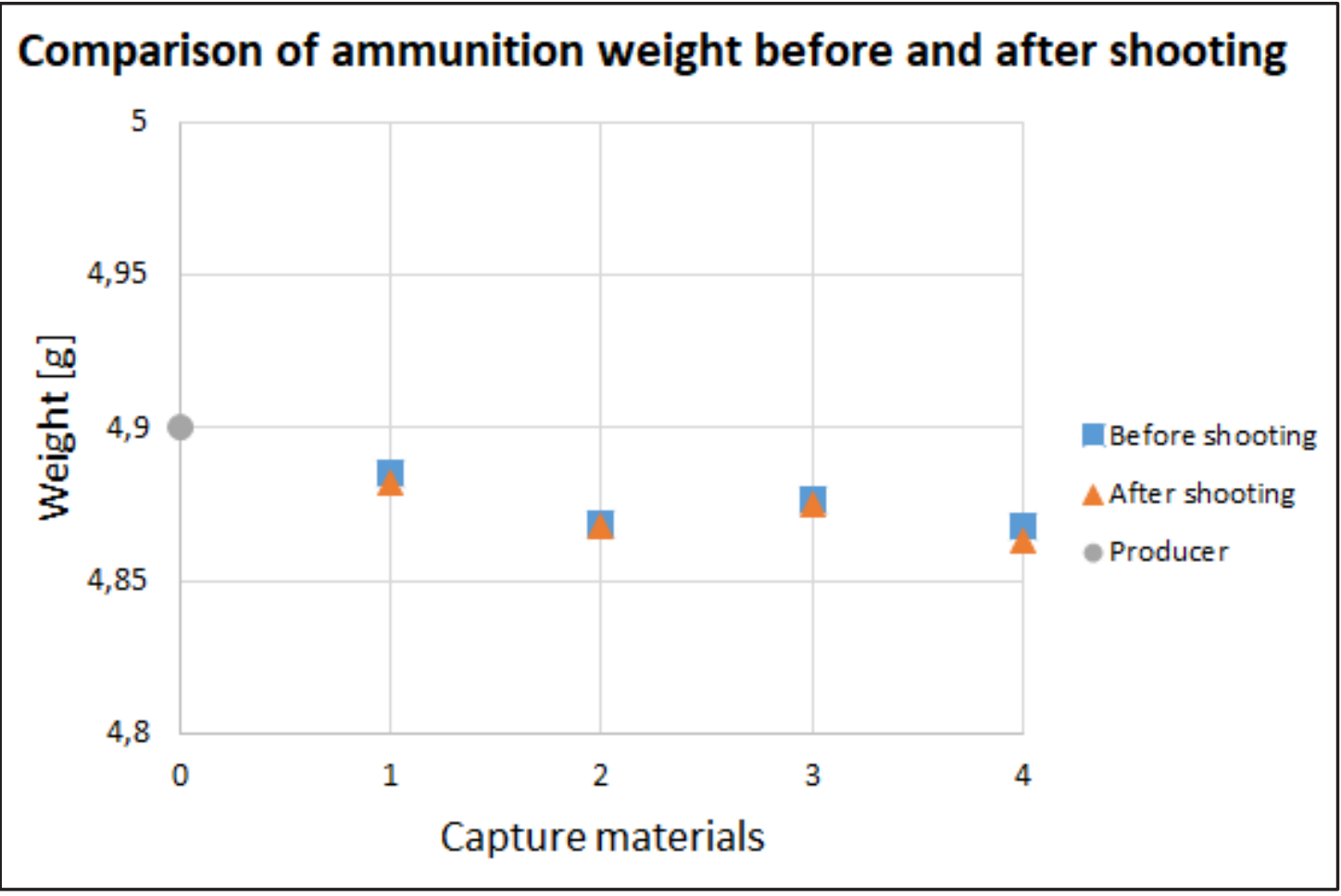

Fig. 6. Comparison of ammunition weight before and after shooting.

Figure 6 shows that a slight loss of weight can occur, but this weight loss is negligible because it is within the measurement error. It can be caused by weight loss after passing the barrel of the firearm or in the catch material or mistake digital scale measurement. For this reason, this is a negligible value and we have not considered it.
We did not get the mass value stated by the manufacturer even before the shot.

\subsection{The effective number of measurements}


In the last experiment, we focused on the effectiveness of the number of measurements. We determined how many times it is necessary to perform repeated measurements to make it effective. As a result, measurement is cheaper and less time-consuming. For this reason, we shooted through one chronograph in succession from 1 to 15 shoots, and individual diaphragm velocities were recorded. Subsequently, the measured data was evaluated. The evaluation was performed by averaging 5 measured data and adding the number gradually, so that the average of all 15 measurements was averaged. Average value and relative measurement error were determined for each mean value. This can be seen in Table 4.

Table 4. The effective number of measurements.

\begin{tabular}{|c|c|c|c|c|c|c|c|c|c|c|c|}
\hline $\begin{array}{c}\begin{array}{c}\text { Number of } \\
\text { measurements }\end{array} \\
\end{array}$ & $\begin{array}{c}\mathbf{V}_{1} \\
{[\mathrm{~m} / \mathrm{s}]}\end{array}$ & $\begin{array}{c}\mathrm{V}_{2} \\
{[\mathrm{~m} / \mathrm{s}]}\end{array}$ & $\begin{array}{c}\mathbf{V}_{3} \\
{[\mathrm{~m} / \mathrm{s}]}\end{array}$ & $\begin{array}{c}\mathrm{V}_{4} \\
{[\mathrm{~m} / \mathrm{s}]}\end{array}$ & $\begin{array}{c}\mathrm{V}_{5} \\
{[\mathrm{~m} / \mathrm{s}]}\end{array}$ & $\begin{array}{c}\mathrm{V}_{6} \\
{[\mathrm{~m} / \mathrm{s}]}\end{array}$ & $\begin{array}{c}\mathrm{V}_{7} \\
{[\mathrm{~m} / \mathrm{s}]}\end{array}$ & $\begin{array}{c}\mathrm{V}_{8} \\
{[\mathrm{~m} / \mathrm{s}]}\end{array}$ & $\begin{array}{c}\mathbf{V}_{9} \\
{[\mathrm{~m} / \mathrm{s}]}\end{array}$ & $\begin{array}{c}\mathrm{V}_{10} \\
{[\mathrm{~m} / \mathrm{s}]}\end{array}$ & $\begin{array}{c}\mathrm{V}_{11} \\
{[\mathrm{~m} / \mathrm{s}]}\end{array}$ \\
\hline 1 & 102 & 102 & 102 & 102 & 102 & 102 & 102 & 102 & 102 & 102 & 102 \\
\hline 2 & 102 & 102 & 102 & 102 & 102 & 102 & 102 & 102 & 102 & 102 & 102 \\
\hline 3 & 99 & 99 & 99 & 99 & 99 & 99 & 99 & 99 & 99 & 99 & 99 \\
\hline 4 & 100 & 100 & 100 & 100 & 100 & 100 & 100 & 100 & 100 & 100 & 100 \\
\hline 5 & 102 & 102 & 102 & 102 & 102 & 102 & 102 & 102 & 102 & 102 & 102 \\
\hline 6 & & 103 & 103 & 103 & 103 & 103 & 103 & 103 & 103 & 103 & 103 \\
\hline 7 & & & 101 & 101 & 101 & 101 & 101 & 101 & 101 & 101 & 101 \\
\hline 8 & & & & 100 & 100 & 100 & 100 & 100 & 100 & 100 & 100 \\
\hline 9 & & & & & 103 & 103 & 103 & 103 & 103 & 103 & 103 \\
\hline 10 & & & & & & 96 & 96 & 96 & 96 & 96 & 96 \\
\hline 11 & & & & & & & 101 & 101 & 101 & 101 & 101 \\
\hline 12 & & & & & & & & 100 & 100 & 100 & 100 \\
\hline 13 & & & & & & & & & 100 & 100 & 100 \\
\hline 14 & & & & & & & & & & 100 & 100 \\
\hline 15 & & & & & & & & & & & 105 \\
\hline Mean & 101.1 & 101.3 & 101.3 & 101.1 & 101.3 & 100.8 & 100.8 & 100.8 & 100.7 & 100.6 & 100.9 \\
\hline $\begin{array}{l}\text { Standard } \\
\text { deviation }\end{array}$ & 1.41 & 1.51 & 1.38 & 1.36 & 1.41 & 2.15 & 2.04 & 1.96 & 1.89 & 1.82 & 2.09 \\
\hline
\end{tabular}

In comparison to 15 measurements and fewer, it was found that it is not important to have as many measurements because the same results can be achieved even with fewer measurements. Therefore, the most appropriate number of measurements for the following experiment was selected (green column in table 4) to save time, money and material. The measurements are shown in Figure 7, which is made from Table 4. It is clear that the number of measurements we have selected is appropriate and the remaining ones are within the measurement error.

\section{Determination of the number of measurements}

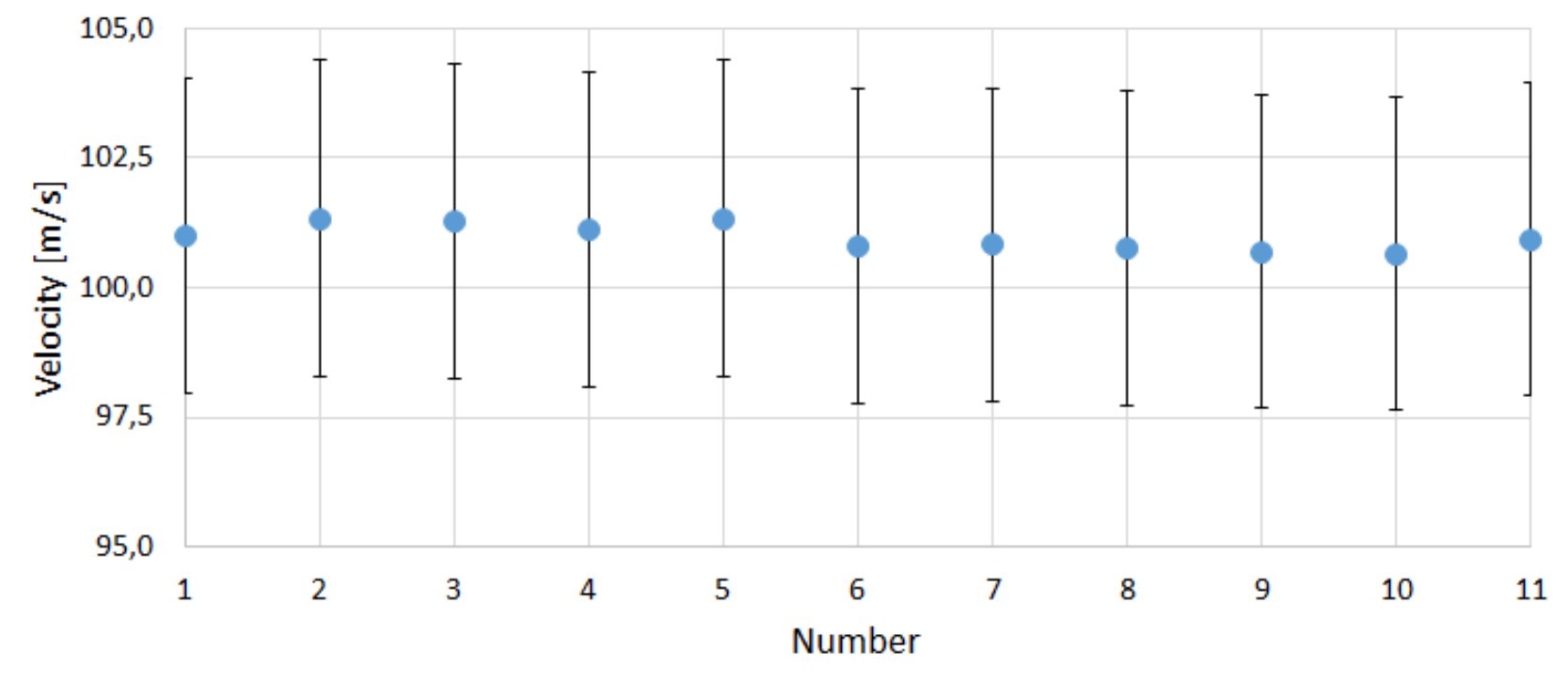

Fig.

7. Determination

of

the

number 
As mentioned above, a green column corresponding to 6 measurements was selected. Because neither in one of the remaining measurements there are not completely balanced bullet velocities and there are anomalies. This then determines on average their average value, which may not be more accurate, but is affected by the anomalies. Any number of measurements is appropriate because of none of the measurements outliers. All counts are within the errors of other measurements. Therefore, we have selected the second lowest number of measurements for the reasons described above.

\section{Conclusion}

This paper described the significance of the experiments and also the benefits for future research. We dealt with the individual descriptions of the experiments we performed. As a part of the individual experiment descriptions, the measured data presented in the clear tables were supplemented by graphical results. Also, with all the data, there was a brief word assessment and a justification of what came out. In future research, our intention is to find out if there is any coefficient that can predict the effect of our "safe" firearms in comparison with "dangerous" firearms. The main advantage of the measurements is the preparation for future research. From this, we have found that the chronograph is sufficient for our purposes and that there is no abrasion in the barrel of the firearm. This is important for the future determination of the wounding potential of weapons of category D. The latest finding in this paper was that the minimal number of measurements is 6 , which is sufficient for the relevant measurement results. A higher number of measurements can increase measurement accuracy, but also increases the measurement error. A compromise of all results was the number of measurements 6 .

This work was supported by Internal Grant Agency of Tomas Bata University in Zlin under the project No. IGA/FAI/2018/014.

\section{References}

1. X. Liu, M. Li, X. Li, X. Deng, X. Zhang, Y. Yan, Y. Liu and X. Chen, J. of Mat. Sc., 53, 7357-7371 (2018)

2. Y. H. Shin, J. H. Chung and J. H. Kim, Int. J. of Nav. Arch. and Oc. Eng., (2018)

3. L-C. Alil, M. Arrigoni, S. Badea, R. Ginghina, L-C. Matache and $P$. Mostovykh, Ex. Pol. Lett., 12, 491-504 (2018)

4. M. J. N. Jacobs, J. L. J. Van Dingenen, J. of Mat. Sc., 36, 3137-3142 (2001)

5. Q. Ma and L. D. Peel, Com. Str., 192, 310316 (2018)
6. P. K. Stefanopoulos, G. F. Hadjigeorgiou, K. Filippakis and D. Gyftokostas, J. of AC. D., 3, 178-185 (2014)

7. R. F. Alban, M. Nuño, A. Ko, G. Barmpares, A. V. Lewis and D. R. Margulies, J. of Sur. Res., 221, 135-142 (2018)

8. M. Mikulicova, M. Gracla, M. Ficek and A. Kuncar, Int. Con. on Mil. Tech. (ICMT), 66-69 (2017) 